\title{
Is the Goutallier grade of multifidus fat infiltration associated with adjacent-segment degeneration after lumbar spinal fusion?
}

\author{
Ping-Guo Duan, MD, ${ }^{1,3}$ Praveen V. Mummaneni, MD, ${ }^{1}$ Jeremy M. V. Guinn, BS, ${ }^{1}$ Joshua Rivera, BA, ${ }^{1}$ \\ Sigurd H. Berven, MD, ${ }^{2}$ and Dean Chou, MD ${ }^{1}$
}

Departments of ${ }^{1}$ Neurological Surgery and ${ }^{2}$ Orthopaedic Surgery, University of California, San Francisco, California; and
${ }^{3}$ Department of Orthopaedic Surgery, The First Affiliated Hospital of Nanchang University, Nanchang, China

OBJECTIVE The aim of this study was to investigate whether fat infiltration of the lumbar multifidus (LM) muscle affects revision surgery rates for adjacent-segment degeneration (ASD) after L4-5 transforaminal lumbar interbody fusion (TLIF) for degenerative spondylolisthesis.

METHODS A total of 178 patients undergoing single-level L4-5 TLIF for spondylolisthesis (2006 to 2016) were retrospectively analyzed. Inclusion criteria were a minimum 2-year follow-up, preoperative MR images and radiographs, and single-level L4-5 TLIF for degenerative spondylolisthesis. Twenty-three patients underwent revision surgery for ASD during the follow-up. Another 23 patients without ASD were matched with the patients with ASD. Demographic data, Roussouly curvature type, and spinopelvic parameter data were collected. The fat infiltration of the LM muscle (L3, L4, and L5) was evaluated on preoperative MRI using the Goutallier classification system.

RESULTS A total of 46 patients were evaluated. There were no differences in age, sex, BMI, or spinopelvic parameters with regard to patients with and those without ASD $(p>0.05)$. Fat infiltration of the LM was significantly greater in the patients with ASD than in those without ASD $(p=0.029)$. Fat infiltration was most significant at $L 3$ in patients with ASD than in patients without ASD $(p=0.017)$. At $L 4$ and $L 5$, there was an increasing trend of fat infiltration in the patients with ASD than in those without ASD, but the difference was not statistically significant ( $p=0.354$ for $L 4$ and $p=0.077$ for $L 5$ ).

CONCLUSIONS Fat infiltration of the LM may be associated with ASD after L4-5 TLIF for spondylolisthesis. Fat infiltration at L3 may also be associated with ASD at L3-4 after L4-5 TLIF.

https://thejns.org/doi/abs/10.3171/2020.6.SPINE20238

KEYWORDS lumbar multifidus; fat infiltration; Goutallier classification; adjacent-segment degeneration; transforaminal lumbar interbody fusion; TLIF; spondylolisthesis; degenerative

$\mathrm{T}$ HE lumbar multifidus (LM) muscles are important for providing lumbar stability; they act as dynamic stabilizers of the lumbar spine by reinforcing lumbar lordosis and resisting lumbar flexion., ${ }^{1,2}$ It has been hypothesized that dysfunction of the low-back muscles can result from fat infiltration of the $\mathrm{LM},{ }^{3}$ reflecting muscle atrophy and degeneration. ${ }^{4}$ Whole-body imaging studies have shown that the LM muscles in spine patients have significant levels of muscle loss and fatty atrophic changes compared with healthy controls. ${ }^{4-7}$ Fat infiltration in the LM is also strongly associated with low-back pain in adults. ${ }^{7,8}$ Low-back pain can not only lead to dysfunction but also result in fat infiltration of the LM muscle as a consequence of muscular atrophy due to underuse. ${ }^{3}$ De- generative conditions of the spine may not only involve the discs and facets but also the paraspinal musculature, ${ }^{4}$ and degeneration of the discs and facets may also precipitate paraspinal muscle degeneration..$^{4,9}$

Fat infiltration of the LM muscle has historically been evaluated using MRI ${ }^{10-12}$ Studies have shown that MRI of the LM muscles in patients with low-back pain reveals a significantly higher fat content than that found in asymptomatic controls. ${ }^{10}$ The Goutallier classification system, first reported in 1994, is a grading system that classifies muscles in the shoulder by the amount of fat within it. ${ }^{13}$ However, this classification system has been used to quantify the degree of fat infiltration in the lumbar paraspinal muscles. ${ }^{11,12}$ Battaglia et al. investigated the reliability of

ABBREVIATIONS ASD = adjacent-segment degeneration; CSA = cross-sectional area; LM = lumbar multifidus; TLIF = transforaminal lumbar interbody fusion.

SUBMITTED February 19, 2020. ACCEPTED June 16, 2020.

INCLUDE WHEN CITING Published online October 30, 2020; DOI: 10.3171/2020.6.SPINE20238. 
the Goutallier classification system for grading muscle fatty degeneration in the LM muscle using MRI, and they showed that this system was reliable in grading LM muscle fat infiltration. ${ }^{11}$ In addition, the amount of fat infiltration graded according to the Goutallier classification has been shown to be associated with disc degeneration. ${ }^{12}$

We hypothesize that fat infiltration of the LM muscle may be associated with adjacent-segment degeneration (ASD) after lumbar fusion. We evaluated revision surgery rates for ASD in patients undergoing single-level L4-5 TLIF for spondylolisthesis and the correlation with fat infiltration by the Goutallier classification.

\section{Methods}

\section{Sample Collection}

A total of 178 patients who underwent single-level transforaminal lumbar interbody fusion (TLIF) for L4-5 spondylolisthesis at the University of California, San Francisco, from 2006 to 2016, were retrospectively analyzed after receiving institutional approval. Inclusion criteria were a minimum 2-year follow-up, single-level L4-5 TLIF for degenerative spondylolisthesis (including lysis), preoperative MRI clear enough to grade fat infiltration, and preoperative 3-foot standing radiographs to evaluate the Roussouly curvature type and spinopelvic parameters. Exclusion criteria were tumor, infection, fusion of $>1$ level, non-TLIF fusions, scoliosis of more than $10^{\circ}$, and TLIF at fusion levels other than L4-5. Patients were also excluded if their adjacent segment exhibited severe preoperative degeneration with $\geq 50 \%$ of disc height loss, $3 \mathrm{~mm}$ or greater listhesis at L3-4, $5^{\circ}$ or more of angulation on flexion and extension radiographs, or grade III or higher Pfirrmann degeneration on sagittal T2-weighted MRI. ${ }^{14}$

\section{Goutallier Classification}

Routine preoperative MR images were obtained. Images were positioned from the midsagittal plane tangentially to the posterior caudal corner of the upper vertebral body and perpendicularly to the surface of the lumbar muscles as illustrated in Fig. 1. Fat infiltration at the three lower segments (L3, L4, and L5) was evaluated preoperatively on axial T1-weighted MR images by three investigators using the Goutallier classification. ${ }^{12,13}$ One attending spine surgeon and two clinical researchers were fully trained on the Goutallier classification method, and they were allocated cases at random without knowledge of whether the patient had ASD. The grades of the LM muscle were defined as follows: grade 0, no intramuscular fat; grade 1, some fatty streaks present; grade 2, fat is evident but less than muscle tissue; grade 3 , the amount of fat is equal to the amount of muscle; and grade 4, more fat than muscle tissue (Fig. 2).

\section{Spinopelvic Parameters}

Three-foot standing lateral radiographs were obtained preoperatively, immediately postoperatively, and at the last clinical follow-up. Radiographic analyses included measurements of pelvic incidence, sacral slope, pelvic tilt, lumbar lordosis, and pelvic incidence minus lumbar lordosis mismatch. After the ASD cohort was identified,

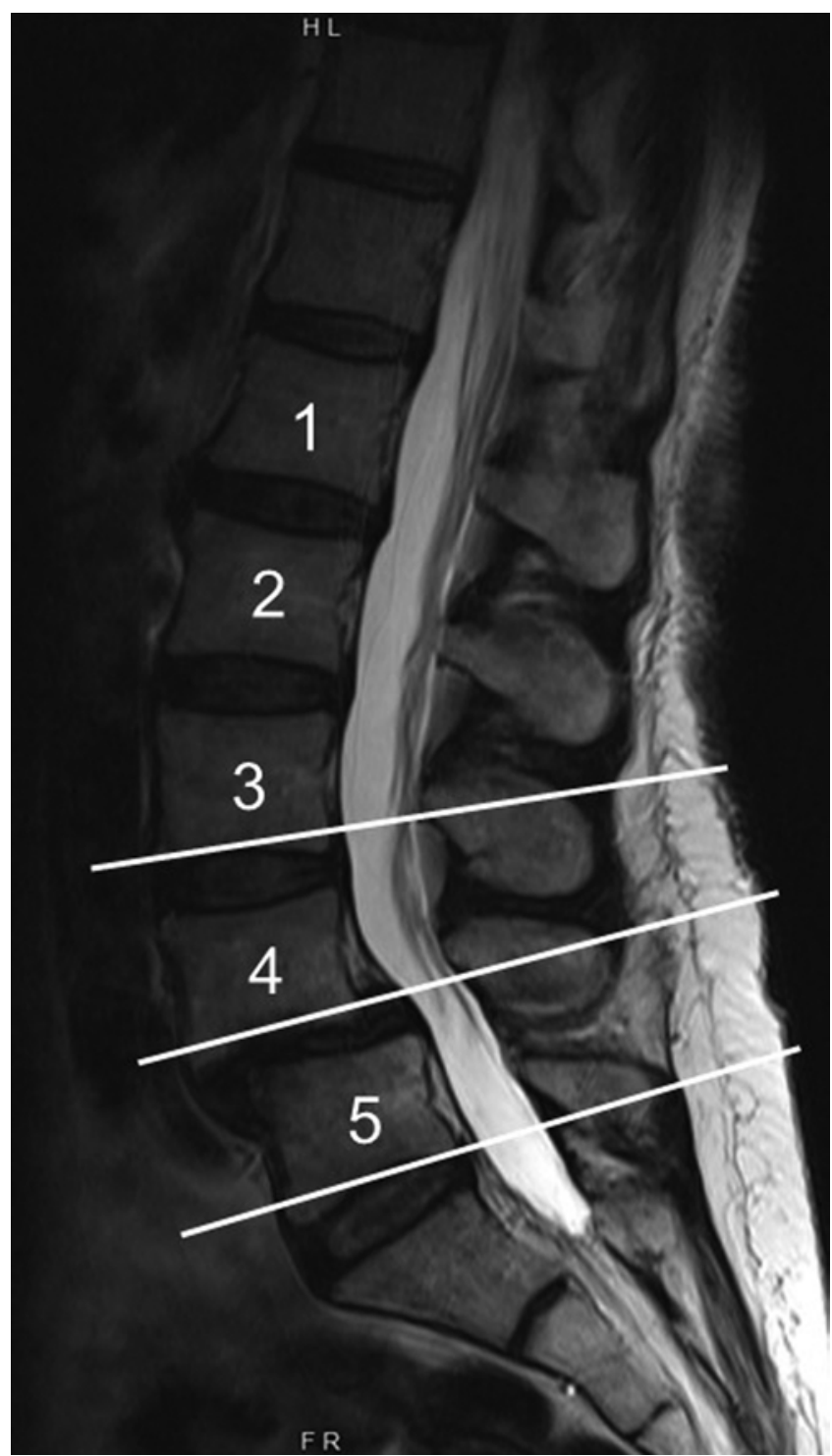

FIG. 1. Sagittal correlation of the location of the axial LM measurements.

spinopelvic parameters were measured. Because spinopelvic parameters have been associated with ASD, controls with the same spinopelvic parameters were identified as the control group. An equal number of patients without ASD and the same spinopelvic parameters were chosen as the control group.

\section{Surgical Technique}

After prone positioning, the spine is exposed, and the surgical level is confirmed with imaging. Pedicle screws are placed either with stereotactic navigation or by fluoroscopy-guided insertion. A total facectomy is then performed, usually on the most severely stenotic side, and Kambin's triangle is accessed. Disc preparation is performed, and endplate cartilage is removed. A cage of an appropriate size (expandable titanium, static titanium, or static polyetheretherketone) is filled with the surgeon's choice of graft (local autograft, iliac crest autograft, al- 


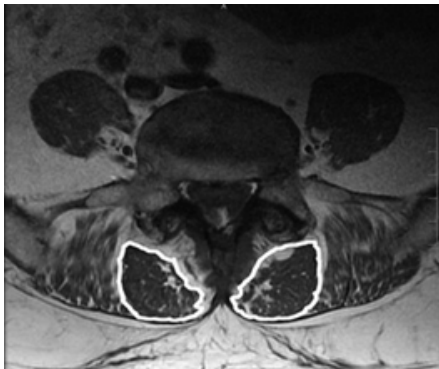

LM

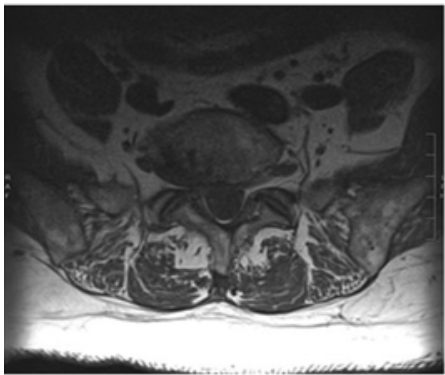

Grade 2

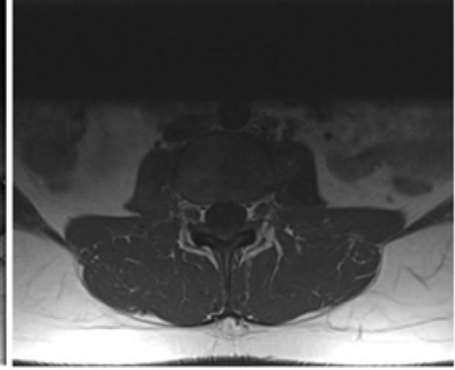

Grade 0

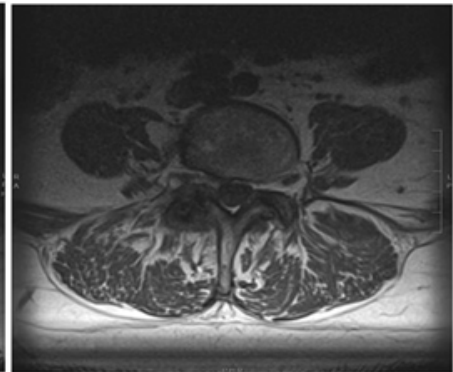

Grade 3

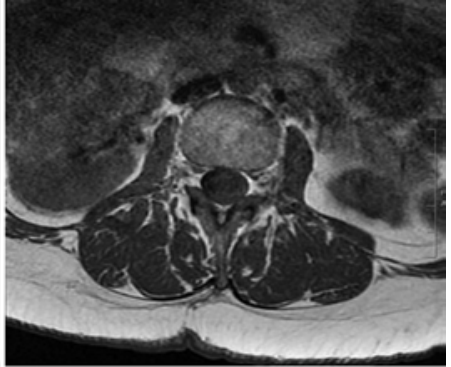

Grade 1

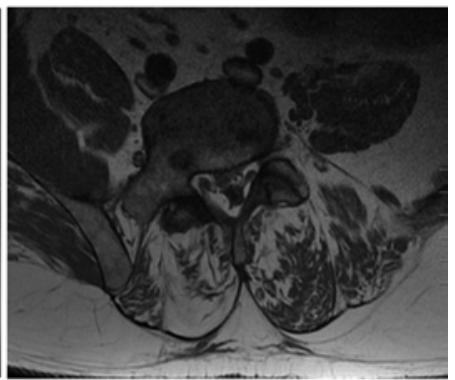

Grade 4

FIG. 2. Goutallier classification of fat infiltration of the LM muscle (outlined in white) on axial T1-weighted MR images. The grades were as follows: grade 0 , no intramuscular fat; grade 1, some fat streaks present; grade 2, fat is evident but less than muscle tissue; grade 3 , the amount of fat is equal to the amount of muscle; grade 4 , more fat than muscle tissue.

lograft, or bone morphogenetic protein), and graft material is placed into the disc space itself. The cage is placed under fluoroscopic guidance, the rods and set screws are placed, graft material is placed laterally for posterolateral fusion, and the wound is closed in layers.

\section{Statistical Analysis}

The data were analyzed using SPSS (version 17.0, SPSS Inc.). Data are reported as means with standard deviations. Comparison between continuous values of two groups was done using independent-samples t-tests. Categorical variables were compared using Fisher's exact test. Statistical significance was noted with $\mathrm{p}<0.05$.

\section{Results}

Of the 178 patients, 23 patients underwent revision surgery for ASD by the last follow-up after TLIF. Twentythree patients without ASD were matched by spinopelvic parameters and used as controls. A total of 46 patients were evaluated (18 males and 28 females, mean age 61.41 \pm 10.46 years). The mean follow-up was $40.01 \pm 18.42$ months (range 24-131 months). Of the entire cohort of 178 patients, 23 patients (12.92\%) underwent revision surgery for ASD in the follow-up period, with 13 patients (7.3\%) undergoing revision at L3-4, 4 patients $(2.25 \%)$ at L5S1, and 6 patients (3.37\%) at both L3-4 and L5-S1. There were no differences in age, sex, BMI, Roussouly type, and spinopelvic parameters with regard to patients with and without ASD ( $p>0.05$; Table 1).

ASD and non-ASD patients were then subdivided, and the total number of levels within each Goutallier grade from L3 to L5 was calculated. Goutallier grade 1
TABLE 1. Patient demographics

\begin{tabular}{|c|c|c|c|}
\hline & $\operatorname{ASD}(n=23)$ & No $\operatorname{ASD}(n=23)$ & $\mathrm{p}$ Value \\
\hline Mean age, yrs & $63.45 \pm 9.0$ & $59.36 \pm 11.92$ & 0.210 \\
\hline F/M sex & $12: 11$ & $16: 7$ & 0.365 \\
\hline Mean BMI & $29.01 \pm 5.31$ & $27.41 \pm 6.38$ & 0.391 \\
\hline $\begin{array}{l}\text { Goutallier classification } \\
\text { (no. of levels) }\end{array}$ & & & $0.029^{*}$ \\
\hline Grade 0 & 0 & 3 & \\
\hline Grade 1 & 13 & 19 & \\
\hline Grade 2 & 38 & 37 & \\
\hline Grade 3 & 12 & 10 & \\
\hline Grade 4 & 6 & 0 & \\
\hline \multicolumn{4}{|l|}{$\begin{array}{l}\text { Mean spinopelvic } \\
\text { parameters, }^{\circ}\end{array}$} \\
\hline PI & $59.09 \pm 10.86$ & $58.36 \pm 10.82$ & 0.825 \\
\hline PT & $21.27 \pm 7.99$ & $22.32 \pm 7.43$ & 0.655 \\
\hline SS & $37.82 \pm 8.94$ & $36.04 \pm 9.24$ & 0.521 \\
\hline $\mathrm{LL}$ & $56.41 \pm 11.56$ & $51.18 \pm 12.69$ & 0.161 \\
\hline PI-LL & $9.36 \pm 8.62$ & $10.09 \pm 9.03$ & 0.786 \\
\hline \multicolumn{4}{|l|}{ Roussouly classification } \\
\hline Type 1 & 1 & 1 & \\
\hline Type 2 & 11 & 11 & \\
\hline Type 3 & 6 & 6 & \\
\hline Type 4 & 5 & 5 & \\
\hline
\end{tabular}

$\mathrm{LL}=$ lumbar lordosis; $\mathrm{PI}=$ pelvic incidence; $\mathrm{PI}-\mathrm{LL}=\mathrm{PI}$ minus $\mathrm{LL} ; \mathrm{PT}=$ pelvic tilt; SS = sagittal slope.

Values represent the number of patients or mean $\pm S D$ unless stated otherwise.

${ }^{*} p<0.05$ 
TABLE 2. Goutallier grades of the LM muscle from L3 to L5 in patients with $(n=23)$ and patients without $(n=23)$ ASD

\begin{tabular}{crrrrrr}
\hline & \multicolumn{7}{c}{ No. of Levels } & \\
\cline { 2 - 5 } & Grade 0 & Grade 1 & Grade 2 & Grade 3 & Grade 4 & p Value \\
\hline L3 & & & & & & \\
\hline ASD & 0 & 6 & 16 & 1 & 0 & \\
\hline No ASD & 3 & 12 & 7 & 1 & 0 & \\
\hline Total & 3 & 18 & 23 & 2 & 0 & $0.017^{*}$ \\
\hline L4 & & & & & & \\
\hline ASD & 0 & 5 & 11 & 6 & 1 & \\
\hline No ASD & 0 & 6 & 15 & 2 & 0 & \\
\hline Total & 0 & 11 & 26 & 8 & 1 & 0.354 \\
\hline L5 & & & & & & \\
\hline ASD & 0 & 2 & 11 & 5 & 5 & \\
\hline No ASD & 0 & 1 & 15 & 7 & 0 & \\
\hline Total & 0 & 3 & 26 & 12 & 5 & 0.077 \\
\hline
\end{tabular}

${ }^{*} p<0.05$, Fisher's exact test.

was observed at 13 and 19 levels with and without ASD, respectively; grade 2 was observed in 38 and 37 levels, respectively; grade 3 was observed in 12 and 10 levels, respectively; and grade 4 was observed in 6 and 0 levels, respectively $(p=0.029)$ (Table 1$)$. For grade 0 , there were 3 patients without ASD, but there were no grade 0 patients with ASD. The inter- and intrarater reliability were calculated as 0.800 and 0.834 , respectively.

When subdivided by level, fat infiltration was more significant at L3 in patients with ASD than in patients without ASD ( $p=0.017)$. At L4 and L5, the difference was not statistically significant $(p=0.354$ for L4 and $p=0.077$ for L5) (Table 2).

\section{Discussion}

Paraspinal muscle degeneration progresses with age and includes a reduction in muscle size and infiltration of fatty degeneration within the muscle. ${ }^{4}$ There is a growing body of evidence showing an association between paraspinal muscle degeneration and lumbar spine issues. . $^{1,5,6,8,15}$ Recently, studies have suggested that both the paraspinal muscle cross-sectional area (CSA) and fat infiltration are associated with low-back pain, radiculopathy, and spinal stenosis. ${ }^{715-17}$ However, other studies have shown the opposite result: that muscle CSA was not associated with spinal degeneration. ${ }^{6,18}$ Some reports have noted that fat infiltration is a better reflection of muscle degeneration compared with the CSA..$^{15,19}$ Other studies have reported that paraspinal muscle damage during lumbar surgery may be one of the most important factors that causes atrophy or fat infiltration of the muscles. ${ }^{20,21}$ In addition, studies have validated the measurement of LM muscle fat infiltration as seen on MRI using the Goutallier classification system, reflecting that this is a reliable system to grade LM fatty degeneration. ${ }^{11,12}$

We found that LM fat infiltration at L3 to L5 was greater in ASD patients than in patients without ASD ( $p$ $=0.029)$ (Table 1). Another interesting point is that the fat infiltration was more significant at L3 in patients with ASD than in those without ASD ( $p=0.017)$ (Table 2). The results indicate that fat infiltration of the LM may contribute to ASD after L4-5 TLIF for spondylolisthesis, especially at L3-4. We found that revision surgery for ASD at the upper adjacent segment (L3-4) was nearly 3 times that of the lower adjacent segment (L5-S1). This finding suggests that the cephalad level is at higher risk for ASD, which is consistent with previous reports. ${ }^{24,25}$ The reason may be that there is increased stress at the cephalad adjacent segment because the L5-S1 ligamentous complex is very tough and strong. ${ }^{26}$ The increased fat infiltration of the LM at L3 may be another reason. ${ }^{27}$ The higher incidence of L3-4 ASD is consistent with the increased fat infiltration of the LM muscles at L3 ( $p<0.05)$, but this correlation was not seen with fat infiltration of the L5 LM muscle and ASD ( $p$ >0.05).

There are a few studies that have investigated the association of spinal disorders and fat infiltration of the LM, erector spinae (ES), and psoas muscles., ${ }^{4,28}$ Fat infiltration of both the LM and ES was associated with reduced intervertebral disc height and an increased risk of Modic changes. Unlike the fat infiltration of the posterior paraspinal muscles, fatty degeneration in the psoas was minimal. ${ }^{4}$ In particular, Teichtahl et al. reported that the high fat content of the ES was significantly associated with Modic changes. ${ }^{18}$ Moreover, a large number of studies have demonstrated that fat infiltration in the LM muscles is strongly associated with low-back and leg pain or poor functional performance of the lumbar spine. $5,7,8,17,18,27$ Taken together, the findings suggest that fat infiltration of the LM muscle is associated with spinal pathology, and it may be a contributing factor to ASD after fusion surgery. ${ }^{8}$

With regard to cohort matching, the initial parameters chosen to perform the matching were spinopelvic parameters. The main reason spinopelvic parameters were chosen as the main factors for matching is that previous studies have shown that spinopelvic mismatch is associated with ASD. ${ }^{22,23}$ Thus, these were the main control variables that we thought were critical to avoid having a significant confounder for ASD. After patients with matched spinopelvic parameters were identified, baseline demographics such as age, sex, and BMI were analyzed, and they were not found to be statistically different. Thus, not only were spinopelvic parameters matched, but also there were no significant differences between age, sex, and BMI. Although there was a difference in the absolute numbers with regard to sex, the statistical calculation of the differences between cohorts revealed a $p$ value of 0.365 . Thus, there was not a statistically significant difference between the two groups with regard to sex. Although older literature suggested that women are more likely than men to develop ASD, a major confounder in those studies was most likely poor bone quality and not necessarily sex per se. For instance, Etebar and Cahill ${ }^{29}$ reported higher rates of "next segment" degeneration in postmenopausal women; however, they also included the fact that more than $50 \%$ were on bisphosphonates, indicating poor bone health as a confounder in their findings. In addition, more recent studies with more rigorous statistical analyses have shown that sex is generally not a risk factor for ASD. ${ }^{30}$ With re- 
gard to the clinical relevance of this information, patient counseling about the understanding and awareness of the increased fatty infiltration can be implemented as a potential prognostic indicator of needing revision surgery. In the future, if the exact cause of fatty infiltration can be elucidated (deconditioning of the musculature, metabolic syndrome from high fructose consumption, or other underlying metabolic disorder), then an intervention such as physical therapy or dietary changes could be implemented to potentially mitigate the risk of progression.

There are some limitations to this study. The results presented should be considered cautiously because of the small sample size. Although there were 178 patients with a minimum 2-year follow-up, the small number of patients with ASD limits the analytical power of the results. Furthermore, the present study was a single-center retrospective study, and the MRI evaluation only focused on the levels of L3 to L5; the upper lumbar musculature was not evaluated. Thus, other factors could account for the variation on paravertebral muscle fat infiltration. However, we tried to make the two comparison groups as homogeneous as possible, matching for spinopelvic parameters, limiting the surgery type to only L4-5 TLIF, and limiting the diagnosis to only degenerative spondylolisthesis at L4-5. Moreover, we focused on evaluating the fat infiltration of the LM muscle and not the CSA of the muscles. The CSA may be another important factor that was not considered in this study, because previous studies have reported that fat infiltration, not muscle CSA, was associated with pain, disability, and structural abnormalities in the lumbar spine..$^{18}$ Another potential limitation is that paraspinal muscular fat infiltration may be a sign of underlying myopathy. Because of the rare diagnosis of primary myopathy, we were not able to delineate patients who had actual myopathies. Another factor that was not addressed in this study is whether or not adjacent-segment facet violation occurred with pedicle screw placement. However, the historical way to assess for superior facet violation is by grading pedicular screw placement according to the method of Gertzbein and Robbins. ${ }^{31}$ This analysis, however, is performed using a postoperative CT scan, and, unfortunately, not all patients had postoperative CT scans, precluding this analysis. However, all TLIFs were performed by surgeons with more than 15 years of experience each, and, hopefully, the knowledge of avoiding the superior facet would have minimized this incidence. Another limitation to this study is the lack of consideration of other factors that are associated with ASD. Although we had excluded preexisting degenerative cases by MRI, other factors that were not accounted for may also have contributed to ASD. Additional studies are necessary to improve our understanding of the association between paraspinal fat infiltration and ASD or other spinal disorders.

\section{Conclusions}

In patients who underwent revision surgery for ASD after L4-5 TLIF for degenerative spondylolisthesis, fat infiltration of the LM muscle was more significant compared with patients without ASD. Fat infiltration of the LM muscle may be associated with ASD after L4-5 TLIF for spondylolisthesis. Fat infiltration at L3 may also be associated with ASD at L3-4 after L4-5 TLIF.

\section{Acknowledgments}

We are grateful for the financial support from the National Science Foundation of China (grant no. 81401790), Natural Science Foundation of Jiangxi Province (grant no. 20171ACB21057), and Science Research Project of Jiangxi Provincial Department of Education (GJJ160028).

\section{References}

1. Hildebrandt M, Fankhauser G, Meichtry A, Luomajoki H. Correlation between lumbar dysfunction and fat infiltration in lumbar multifidus muscles in patients with low back pain. BMC Musculoskelet Disord. 2017;18(1):12.

2. Panjabi MM. Clinical spinal instability and low back pain. $J$ Electromyogr Kinesiol. 2003;13(4):371-379.

3. Freeman MD, Woodham MA, Woodham AW. The role of the lumbar multifidus in chronic low back pain: a review. $P M R$. 2010;2(2):142-146, quiz 1, 167.

4. Lee SH, Park SW, Kim YB, et al. The fatty degeneration of lumbar paraspinal muscles on computed tomography scan according to age and disc level. Spine J. 2017;17(1):81-87.

5. Shahidi B, Hubbard JC, Gibbons MC, et al. Lumbar multifidus muscle degenerates in individuals with chronic degenerative lumbar spine pathology. J Orthop Res. 2017;35(12): 2700-2706.

6. Shahidi B, Parra CL, Berry DB, et al. Contribution of lumbar spine pathology and age to paraspinal muscle size and fatty infiltration. Spine (Phila Pa 1976). 2017;42(8):616-623.

7. Kjaer P, Bendix T, Sorensen JS, et al. Are MRI-defined fat infiltrations in the multifidus muscles associated with low back pain? BMC Med. 2007;5:2.

8. Kalichman L, Carmeli E, Been E. The association between imaging parameters of the paraspinal muscles, spinal degeneration, and low back pain. Biomed Res Int. 2017;2017: 2562957.

9. Yoshihara K, Shirai Y, Nakayama Y, Uesaka S. Histochemical changes in the multifidus muscle in patients with lumbar intervertebral disc herniation. Spine (Phila Pa 1976). 2001; 26(6):622-626.

10. Mengiardi B, Schmid MR, Boos N, et al. Fat content of lumbar paraspinal muscles in patients with chronic low back pain and in asymptomatic volunteers: quantification with MR spectroscopy. Radiology. 2006;240(3):786-792.

11. Battaglia PJ, Maeda Y, Welk A, et al. Reliability of the Goutallier classification in quantifying muscle fatty degeneration in the lumbar multifidus using magnetic resonance imaging. J Manipulative Physiol Ther. 2014;37(3):190-197.

12. Tamai K, Chen J, Stone M, et al. The evaluation of lumbar paraspinal muscle quantity and quality using the Goutallier classification and lumbar indentation value. Eur Spine J. 2018;27(5):1005-1012.

13. Goutallier D, Postel JM, Bernageau J, et al. Fatty muscle degeneration in cuff ruptures. Pre- and postoperative evaluation by CT scan. Clin Orthop Relat Res. 1994;(304):78-83.

14. Pfirrmann CW, Metzdorf A, Zanetti M, et al. Magnetic resonance classification of lumbar intervertebral disc degeneration. Spine (Phila Pa 1976). 2001;26(17):1873-1878.

15. Urrutia J, Besa P, Lobos D, et al. Lumbar paraspinal muscle fat infiltration is independently associated with sex, age, and inter-vertebral disc degeneration in symptomatic patients. Skeletal Radiol. 2018;47(7):955-961.

16. Fortin M, Lazáry À, Varga PP, Battié MC. Association between paraspinal muscle morphology, clinical symptoms and functional status in patients with lumbar spinal stenosis. Eur Spine J. 2017;26(10):2543-2551. 
17. Kader DF, Wardlaw D, Smith FW. Correlation between the MRI changes in the lumbar multifidus muscles and leg pain. Clin Radiol. 2000;55(2):145-149.

18. Teichtahl AJ, Urquhart DM, Wang Y, et al. Fat infiltration of paraspinal muscles is associated with low back pain, disability, and structural abnormalities in community-based adults. Spine J. 2015;15(7):1593-1601.

19. Ranson CA, Burnett AF, Kerslake R, et al. An investigation into the use of MR imaging to determine the functional cross sectional area of lumbar paraspinal muscles. Eur Spine J. 2006;15(6):764-773.

20. Hyun SJ, Kim YB, Kim YS, et al. Postoperative changes in paraspinal muscle volume: comparison between paramedian interfascial and midline approaches for lumbar fusion. $J \mathrm{Ko}$ rean Med Sci. 2007;22(4):646-651.

21. Suwa H, Hanakita J, Ohshita N, et al. Postoperative changes in paraspinal muscle thickness after various lumbar back surgery procedures. Neurol Med Chir (Tokyo). 2000;40(3): $151-155$.

22. Liang J, Dong Y, Zhao H. Risk factors for predicting symptomatic adjacent segment degeneration requiring surgery in patients after posterior lumbar fusion. J Orthop Surg Res. 2014;9:97.

23. Moreau PE, Ferrero E, Riouallon G, et al. Radiologic adjacent segment degeneration 2 years after lumbar fusion for degenerative spondylolisthesis. Orthop Traumatol Surg Res. 2016;102(6):759-763.

24. Zhong ZM, Deviren V, Tay B, et al. Adjacent segment disease after instrumented fusion for adult lumbar spondylolisthesis: incidence and risk factors. Clin Neurol Neurosurg. 2017;156: 29-34.

25. Okuda S, Nagamoto Y, Matsumoto T, et al. Adjacent segment disease after single segment posterior lumbar interbody fusion for degenerative spondylolisthesis: minimum 10 years follow-up. Spine (Phila Pa 1976). 2018;43(23):E1384-E1388.

26. Akamaru T, Kawahara N, Yoon ST, et al. Adjacent segment motion after a simulated lumbar fusion in different sagittal alignments: a biomechanical analysis. Spine (Phila Pa 1976). 2003;28(14):1560-1566.

27. Battié MC, Niemelainen R, Gibbons LE, Dhillon S. Is leveland side-specific multifidus asymmetry a marker for lumbar disc pathology? Spine J. 2012;12(10):932-939.
28. Pezolato A, de Vasconcelos EE, Defino HL, Nogueira-Barbosa MH. Fat infiltration in the lumbar multifidus and erector spinae muscles in subjects with sway-back posture. Eur Spine J. 2012;21(11):2158-2164.

29. Etebar S, Cahill DW. Risk factors for adjacent-segment failure following lumbar fixation with rigid instrumentation for degenerative instability. J Neurosurg. 1999;90(2 Suppl): $163-169$.

30. Ma Z, Huang S, Sun J, et al. Risk factors for upper adjacent segment degeneration after multi-level posterior lumbar spinal fusion surgery. J Orthop Surg Res. 2019;14(1):89.

31. Gertzbein SD, Robbins SE. Accuracy of pedicular screw placement in vivo. Spine (Phila Pa 1976). 1990;15(1):11-14.

\section{Disclosures}

Dr. Mummaneni: consultant for Globus, DePuy Synthes, and Stryker; direct stock ownership in Spinicity/ISD; honoraria from Spineart; royalties from DePuy Synthes, Thieme Publishers, and Springer Publishers; and non-study-related clinical or research effort from AO Spine and NREF. Dr. Berven: consultant for Green Sun Medical, Globus, Medtronic, Medicrea, and Integrity and royalties from Medtronic and Stryker. Dr. Chou: consultant for Globus and Medtronic and royalties from Globus.

\section{Author Contributions}

Conception and design: Chou. Acquisition of data: Duan, Guinn, Rivera. Analysis and interpretation of data: Duan. Drafting the article: Duan. Critically revising the article: Mummaneni, Berven, Chou. Reviewed submitted version of manuscript: Mummaneni, Berven. Approved the final version of the manuscript on behalf of all authors: Duan. Administrative/technical/material support: Mummaneni, Guinn, Rivera, Berven. Study supervision: Chou.

\section{Correspondence}

Ping-Guo Duan: University of California, San Francisco, CA. pink1198@163.com. 